\title{
Non-traumatic acute paraplegia associated with a CT-guided needle biopsy in a silicotic nodule: A case report
}

\author{
LIYING XU, XUN DING and MEIYAN LIAO \\ Department of Radiology, Zhongnan Hospital of Wuhan University, Wuhan, Hubei 430071, P.R. China
}

Received July 16, 2015; Accepted December 7, 2015

DOI: $10.3892 / \mathrm{mco} .2015 .711$

\begin{abstract}
The present study reports the case of an adult patient with non-traumatic acute paraplegia following a computed tomography (CT)-guided automated cutting needle biopsy (ACNB). Multiple nodules and masses were revealed on performing chest radiography and CT on a 45-year-old man. In order to make a pathological diagnosis, a CT-guided biopsy using an automatic cutting needle was performed. However, $10 \mathrm{~min}$ after the biopsy, a weakness of the lower extremities occurred, and the patient collapsed to the ground, albeit with clear consciousness. Spinal magnetic resonance imaging (MRI) performed subsequently revealed no abnormal findings in the spinal cord. An MRI performed $24 \mathrm{~h}$ later, however, revealed swelling of the thoracic spinal cord and a high-signal-intensity lesion in T2-weighted images at the level of T7, T8 and T9. The patient subsequently received hyperbaric oxygen therapy for a few days, and rehabilitative treatment over the course of a few weeks. At 6 months following the biopsy, the patient was unable to walk, although the patient could stand for $10 \mathrm{~min}$ and defecate independently. Currently, the patient remains active in daily life, in spite of confinement to a wheelchair. The present case study was reported to raise the awareness of the possibility of spinal cord ischemia and acute paraplegia following a CT-guided ACNB of the lungs. The mechanism underlying spinal cord ischemia remains to be fully elucidated, although is thought to be multifactorial, involving air embolism.
\end{abstract}

\section{Introduction}

Computer tomography (CT)-guided automated cutting needle biopsy (ACNB) is a minimally invasive technique, which is an accepted and widely used tool in the diagnosis of pulmonary lesions (1-3). Although considered to be a safe technique, several

Correspondence to: Dr Liying Xu, Department of Radiology, Zhongnan Hospital of Wuhan University, no. 169 Donghu Road, Wuhan, Hubei 430071, P.R. China

E-mail: aying0923@foxmail.com

Key words: CT-guided needle biopsy, complication, paraplegia, spinal cord ischemia, air embolism complications have been associated with the procedure, including the occurrence of pneumothorax, hemorrhaging, air embolism and tumor seeding (4-6). In the present study, a very rare case of non-traumatic acute paraplegia resulting from an ACNB in a patient with silicosis is reported. Although the symptoms of the patient in the present case study were substantially relieved following aggressive treatment, the patient still presented with a poor neurological recovery and permanent deficit. To the best of our knowledge, no previous case report has described paraplegia due to CT-guided ACNB of the lungs.

\section{Case report}

A 45-year-old man was admitted to the Zhongnan Hospital of Wuhan University with a history of productive coughing, which had lasted for a duration of 1 year. Multiple nodules and masses were revealed on performing chest radiography and CT. In order to make a pathological diagnosis, a CT-guided biopsy using an automatic cutting needle (18-gauge; Bard ${ }^{\circledR}$ Maxcore ${ }^{\circledR}$, Bard Biopsy Systems, Tempe, AZ, USA) was required. The largest accessible lung mass was selected to be the targeted area for biopsy, also considering the needle puncture pathway and the direction of the targeted mass. The transthoracic distance to the lung mass was selected to be the shortest, without any evident emphysematous changes or bulla in the pathway of the needle entrance. Subsequently, the patient was placed into the prone position. Limited CT (Somatom Sensation 16; Siemens Healthcare, Erlangen, Germany) scanning was performed to localize the lesion and to document the needle progression to the depth of the target. The puncture point on the skin was labeled. The distance between the targeted mass and the pleura was $20 \mathrm{~mm}$, and the distance between the puncture point and the spine tube was $70 \mathrm{~mm}$ (Fig. 1). Subsequently, the biopsy was performed under aseptic conditions and local anesthesia. A total of three tissue samples were obtained. The tumor tissues were immersed in $10 \%$ formalin, and processed for conventional pathological examination. Immediately following the procedure, the patient coughed up approximately one tablespoon's volume of bloody sputa. A CT scan was performed again to assess the complications of the biopsy. No pneumothorax or bleeding was identified (Fig. 2). The patient exited the CT room by himself. However, $10 \mathrm{~min}$ later, the patient suddenly felt weakness in his lower limbs and fell to the ground with clear consciousness. The patient was 


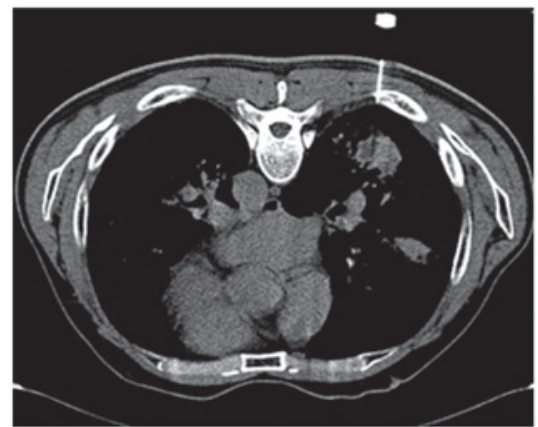

Figure 1. CT-guided lung biopsy of a mass lesion in the right lower lobe. The image also shows the biopsy needle. The distance between the targeted mass and the pleura was $20 \mathrm{~mm}$, and the distance between the puncture point and the spine tube was $70 \mathrm{~mm}$.

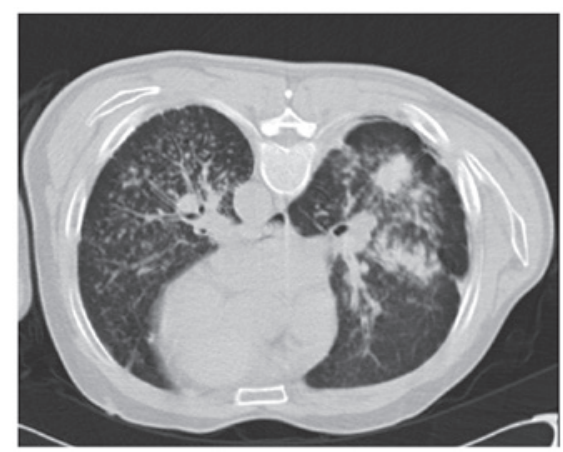

Figure 2. CT imaging following biopsy, revealing a small amount of bleeding in the thoracic cavity, although no pneumothorax was identified.

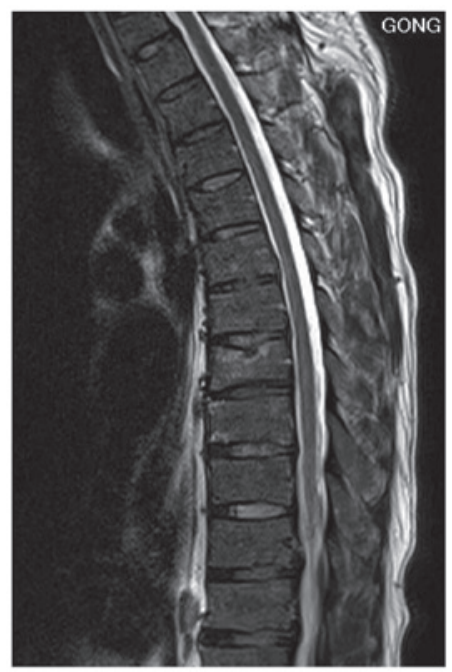

Figure 3. Spinal magnetic resonance imaging after $2 \mathrm{~h}$, revealing no abnormal findings in the spinal cord.

immediately sent to the emergency department close to the CT room. Supplemental 100\% oxygen was administered, and general symptomatic support was provided. A neurological examination revealed a flaccid paraplegia. Hyperesthesia at the level below the T8 dermatome was observed. The anal reflex was absent, and the patient experienced urinary and fecal incontinence. Subsequent spine magnetic resonance imaging (MRI) demonstrated no abnormal findings in the

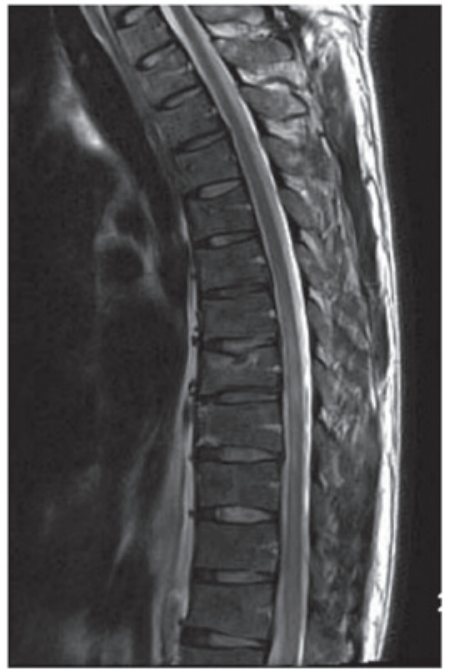

Figure 4 A representative T2-weighted image showing spinal magnetic resonance imaging after $24 \mathrm{~h}$. Swelling of the thoracic spinal cord and a high-signal-intensity lesion in were revealed at the level of T7, T8 and T9.

spinal cord (Fig. 3). An MRI was performed on the subsequent day, which revealed swelling of the thoracic spinal cord; in addition, a high-signal-intensity lesion at the level of T7, T8 and T9 was identified in T2-weighted images (Fig. 4). The patient subsequently received hyperbaric oxygen therapy for a few days, and rehabilitative treatment over a period of a few weeks. The patient made a gradual recovery over 4 months. At 6 months following the biopsy, the patient remained unable to walk, although the patient could stand for $10 \mathrm{~min}$ and defecate independently. At present, the patient remains active in daily life, in spite of confinement to a wheelchair.

\section{Discussion}

The present case report highlights that patients undergoing CT-guided ACNB are susceptible to spinal cord ischemia, which, to the best of our knowledge, has not been previously reported. The mechanism underlying spinal cord ischemia remains to be fully elucidated, although it is considered to be multifactorial, involving air embolism. A plausible hypothesis is that concurrent coughing during the procedure is able to displace the biopsy needle into the large vessel adjacent to the pulmonary lesion, and continuous high intrathoracic pressure may have pushed a large quantity of air into the pulmonary vein, leading to systemic air embolism.

CT-guided lung biopsy is a frequently performed procedure, and is important in the evaluation of solitary and multiple pulmonary nodules. Minor complications commonly (30-60\%) associated with the procedure include pneumothorax of low abundance (17-60\%) and alveolar bleeding (5-30\%) (7). In general, these complications are asymptomatic, and regress spontaneously. Serious complications requiring a specific treatment, including drained pneumothorax, hemopericardium, hemothorax, hemoptysis, intense pain and air embolism (1-8), rarely occur. Air embolism is the most severe complication, although it is among those that occur the least frequently. In a study of 9,783 biopsies, air embolism occurred in six patients, resulting in an incidence rate of $0.06 \%$ (1), 
which also revealed no major difference from the previously reported complication rate.

The present case study has been reported to raise the awareness of the possibility of such a complication arising in CT-guided ACNB of the lungs. Although very rare in occurrence, however, this kind of complication may occur in a patient with interstitial lung disease and emphysema (9), such as in the present case. Although air embolism occurs only very rarely as a complication of percutaneous needle biopsy, physicians should be aware of its occurrence and significance. Air embolism may lead to myocardial infarction, arrhythmia, stroke and mortality. The operators must check their practice, and calculate the risk of complications. The risks should be clearly explained to the patients in order that they may give their informed consent prior to the procedure. Once an air embolism is suspected, the patient should be placed into either the left lateral decubitus or the Trendelenberg position to prevent residual air in the left atrium from entering the cerebral circulation. Supplemental $100 \%$ oxygen should be administered, and general symptomatic support should be provided (10).

\section{References}

1. Tomiyama N, Yasuhara Y, Nakajima Y, Adachi S, Arai Y, Kusumoto M, Eguchi K, Kuriyama K, Sakai F, Noguchi M, et al: CT-guided needle biopsy of lung lesions: A survey of severe complication based on 9783 biopsies in Japan. Eur J Radiol 59: 60-64, 2006
2. Khan MF, Straub R, Moghaddam SR, Maataoui A, Gurung J Wagner TO, Ackermann H, Thalhammer A, Vogl TJ and Jacobi V: Variables affecting the risk of pneumothorax and intrapulmonal hemorrhage in CT-guided transthoracic biopsy. Eur Radiol 18: 1356-1363, 2008.

3. Wu CC, Maher MM and Shepard JA: Complications of CT-guided percutaneous needle biopsy of the chest: Prevention and management. AJR Am J Roentgenol 196: W678-W682, 2011.

4. Nour-Eldin NE, Alsubhi M, Naguib NN, Lehnert T, Emam A, Beeres M, Bodelle B, Koitka K, Vogl TJ and Jacobi V: Risk factor analysis of pulmonary hemorrhage complicating CT-guided lung biopsy in coaxial and non-coaxial core biopsy techniques in 650 patients. Eur J Radiol 83: 1945-1952, 2014.

5. Zhuang YP, Wang HY, Zhang J, Feng Y and Zhang L: Diagnostic accuracy and safety of CT-guided fine needle aspiration biopsy in cavitary pulmonary lesions. Eur J Radiol 82: 182-186, 2013.

6. O'Neill AC, McCarthy C, Ridge CA, Mitchell P, Hanrahan E, Butler M, Keane MP and Dodd JD: Rapid needle-out patient-rollover time after percutaneous CT-guided transtho-racic biopsy of lung nodules: Effect on pneumothorax rate. Radiology 262: 314-319, 2012.

7. Prosch H, Stadler A, Schilling M, Bürklin S, Eisenhuber E, Schober E and Mostbeck G: CT fluoroscopy-guided vs. multislice CT biopsy mode-guided lung biopsies: Accuracy, complications and radiation dose. Eur J Radiol 81: 1029-1033, 2012.

8. Cheung YC, Chang JW, Hsieh JJ, Lin G and Tsai YH: Adequacy and complications of computed tomography-guided core needle biopsy on non-small cell lung cancers for epidermal growth factor receptor mutations demonstration: 18-gauge or 20-gauge biopsy needle. Lung Cancer 67: 166-169, 2010.

9. Heyer CM, Reichelt S, Peters SA, Walther JW, Müller KM and Nicolas V: Computed tomography-navigated transthoracic core biopsy of pulmonary lesions: Which factors affect diagnostic yield and complication rates? Acad Radio 15: 1017-1026, 2008.

10. Klein JS and Zarka MA: Transthoracic needle biopsy. Radiol Clin North Am 38: 235-266, 2000. 